\title{
Estimation and Visualization of Identity- by-Descent within Pedigrees Simplifies Interpretation of Complex Trait Analysis
}

\author{
Elizabeth E. Marchani ${ }^{\mathrm{a}} \quad$ Ellen M. Wijsman ${ }^{\mathrm{a}} \mathrm{b}$ \\ ${ }^{a}$ Division of Medical Genetics, Department of Medicine, and ${ }^{b}$ Department of Biostatistics, University of \\ Washington, Seattle, Wash., USA
}

\section{Key Words}

Inheritance vector $\cdot$ Segregation $\cdot$ Genome scan .

Haplotype $\cdot$ Equivalence class

\begin{abstract}
Linkage analysis identifies markers that appear to be co-inherited with a trait within pedigrees. The inheritance of a chromosomal segment may be probabilistically reconstructed, with missing data complicating inference. Inheritance patterns are further obscured in the analysis of complex traits, where variants in one or more genes may contribute to phenotypic variation within a pedigree. In this case, determining which relatives share a trait variant is not simple. We describe how to represent these patterns of inheritance for marker loci. We summarize how to sample patterns of inheritance consistent with genotypic and pedigree data using gl_auto, available in MORGAN v3.0. We describe identification of classes of equivalent inheritance patterns with the program IBDgraph. We finally provide an example of how these programs may be used to simplify interpretation of linkage analysis of complex traits in general pedigrees. We borrow information across loci in a parametric linkage analysis of a large pedigree. We explore the contribution of each equivalence class to a linkage signal, illustrate estimated pat-
\end{abstract}

terns of identity-by-descent sharing, and identify a haplotype tagging the chromosomal segment driving the linkage signal. Haplotype carriers are more likely to share the linked trait variant, and can be prioritized for subsequent DNA sequencing.

Copyright $\odot 2011$ S. Karger AG, Base

\section{Introduction}

Linkage analysis uses pedigree data to find markers that appear to be co-inherited with a trait-related variant, suggesting close proximity on a chromosome [1-3]. Linkage analysis has been used to successfully identify multiple rare variants in thousands of genes related to Mendelian diseases $[4,5]$. Recent genome-wide scans of sequencing data have used a similar strategy to find genetic variants causing rare Mendelian disease; they looked for rare variants shared identical-by-descent (IBD) among affected relatives [6]. Linkage analyses can also be tailored to find genes influencing complex traits, such as loci altering the penetrance of primary disease variants (e.g. [7]), phenotypic modifiers (e.g. [8]), or quantitative trait loci (e.g. [9]).

\section{KARGER}

(๑) 2011 S. Karger AG, Basel

Fax +4161306 1234

E-Mail karger@karger.ch

www.karger.com
Accessible online at: www.karger.com/hhe
Dr. Ellen M. Wijsman

Division of Medical Genetics

University of Washington

Box 357720, Seattle, WA 98195-7720 (USA)

Tel. +1 206543 8987, E-Mail wijsman@u.washington.edu 


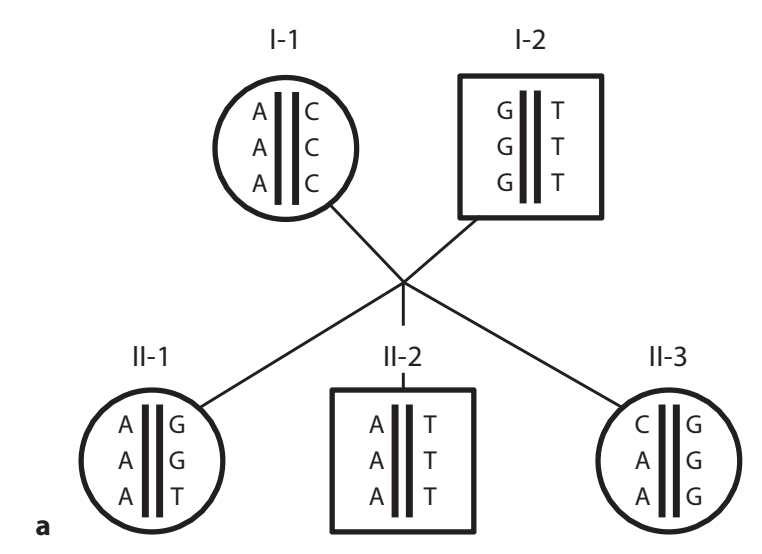

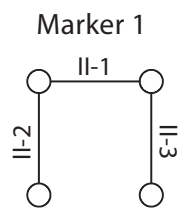

b

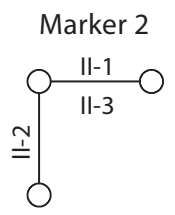

$[0,0,0,1,0,0]$

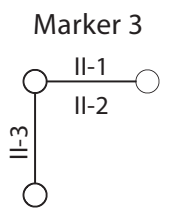

$[0,1,0,1,0,0]$
Fig. 1. Patterns of inheritance at three markers within a pedigree. a Pedigree structure and phased genotypes. Subjects labeled by generation (Roman numerals) and an Arabic numeral. Maternally (paternally) derived alleles are shown on the left (right). b IBD graphs and inheritance vectors (IVs) for each marker. Shared nodes indicate alleles shared IBD. Meioses described by the IV are in the order of phased genotypes from left to right in the second generation: 0 = alleles derived from a parent's mother, $1=$ alleles derived from a parent's father.

The ultimate goal of linkage analysis is to identify genes influencing the phenotype under study. Co-segregation of the candidate locus and the trait acts as important evidence in this search, but can be difficult to detect when studying complex traits. Many common disorders are complex traits, influenced by environmental factors as well as one or more variants in multiple genes. This genetic and allelic heterogeneity has been documented in several, very different phenotypes, including Alzheimer's disease (AD) [10-13], breast and ovarian cancer [14], and familial hypercholesterolemia $[15,16]$. In each case, a simple Mendelian model of inheritance, where one variant co-segregates perfectly with disease status, does not fit the data. Instead, several genetic and environmental variables influence the genotype/phenotype probabilities [3]. It is quite possible for multiple genetic variants influencing the trait to segregate within a family, perhaps none of which is sufficient alone to cause disease. This makes the relationship between variant and phenotype difficult to determine, especially for common traits. However, large pedigrees improve the power of linkage analyses [17] and have enough copies of a rare variant to determine that it is influential $[18,19]$. This improved power has been shown in successful genome scans for modifier loci with relatively small sample sizes [7,20,21]. A single large pedigree is also expected to segregate fewer trait-related variants than a larger collection of smaller pedigrees [3], simplifying the relationship between genotype and phenotype.

Pedigrees with genotype data, combined with Mendel's laws of segregation, allow the probabilistic reconstruction of the inheritance of a chromosomal segment through a pedigree. Inheritance vectors (IVs) trace the path connecting alleles to their maternal or paternal origin at each generation of the pedigree. IVs can be used to define chromosomal segments or haplotypes, with boundaries defined by recombination events [22-24]. Here, we will review a new method to estimate IBD segments within a pedigree, and demonstrate how to find the IBD segment driving a linkage signal. Specifically, we will describe (1) the new program gl_auto in MORGAN v3.0 [25] which collects and saves samples of IVs consistent with the observed pedigree and genotype data [22], and (2) how to summarize gl_auto output using IBDgraph, which collapses the sample into equivalence classes of realizations with identical patterns of IBD sharing. We will then use an example to show how patterns of IBD sharing within a pedigree can be used to identify the chromosomal segment driving a linkage signal and which relatives carry the segment IBD. Additional experiments are required to determine whether these subjects share a true trait locus within that region [23]. Finally, we will discuss other possible uses for IVs.

\section{Representing Marker Inheritance}

Figure 1 illustrates three ways to represent marker inheritance through a pedigree. Figure la is the pedigree of a nuclear family. Within the circle (female) or square (male) representing an individual are the phased genotypes at three neighboring and perfectly informative marker loci. If we care only about IBD sharing and not marker alleles, we could replace allele labels with founder genome labels (FGLs). For example, the A alleles could each be replaced with FGL $=1$; the $\mathrm{C}$ alleles could be replaced with $\mathrm{FGL}=2$, and so on. FGLs are unique labels 
for each founder chromosome in the pedigree; relatives who share an FGL share that marker allele IBD [22].

Figure $1 \mathrm{~b}$ illustrates both IBD graphs and IVs for each marker. In an IBD graph, or descent graph [24], each node represents a unique founder chromosome, and lines connect founder chromosomes inherited together [22]. IBD graphs illustrate only the pattern of IBD sharing among non-founders; it does not matter what specific FGL is associated with each node. For example, individuals II-2 and II-3 share a different allele IBD with II-1 at marker 1, while individual II-3 shares both alleles IBD with II-1 at marker 2. Beneath each IBD graph is the corresponding IV, represented as an array $[22,24,26]$. Each IV is pedigree and marker specific, with an element for each meiosis. We use a value of 0 (1) to indicate a locus inherited from the parent's maternally (paternally) derived chromosome [27]. The meioses are ordered from left to right in the offspring generation: e.g. element 1 of marker 1's IV indicates that individual II-1 inherited her mother's maternally derived allele, element 2 indicates that individual II-1 inherited her father's maternally derived allele, element 3 indicates individual II-2 inherited his mother's maternally derived allele, etc. We infer a recombination event when alleles at neighboring loci are inherited in a combination that differs from that observed in the parent, indicated by an IV switching states from maternal to paternal, or vice versa. Comparing IVs across markers, we infer a recombination event between markers 1 and 2 for meiosis 5, and recombination events between markers 2 and 3 for meiosis 2 .

Inference of these inheritance patterns is straightforward when perfectly informative marker data are available. Unfortunately, this is rarely the case with real data. The theory [1] and algorithms [26,28] for using these inheritance patterns in the context of likelihood analysis are well established. When using data from multiple markers in a multipoint linkage analysis, we gain greater statistical power for detecting linkage and fewer false positive signals [17]. Similarly, a gain in power, precision, and consistency across analyses with fewer false positives is achieved when we analyze large pedigrees $[17,18]$. Algorithms exist that are well suited for analyzing large pedigrees [28] or many markers [26] with exact computation, but not both [29]. Analysis of large pedigrees with many markers is computationally intense, often prohibitively so.

Advances in computation and analytical techniques now permit the use of more complicated disease models and the analysis of larger pedigrees. Markov chain Monte Carlo (MCMC) techniques are used to estimate linkage statistics when the size of the pedigree and number of markers are too large for exact computation to be practical. Instead of cataloging every possible pattern of genotype and/or phenotype data consistent with the observed data, MCMC methods sample from the distribution of possibilities conditional on the observed data. Sophisticated linkage analysis packages, such as SimWalk [30, 31], MORGAN [32-36], and Loki [35, 37, 38] use MCMC methods to combine population allele frequencies and marker positions with pedigree structure and trait data to create and collect thousands of samples of complete marker and trait-locus data $[24,37,39,40]$. The expected value of the linkage statistic is computed using the average across these thousands of data sets. A recently released program called gl_auto [25] is the only MCMC program to report the entire sample of IVs [29].

\section{gl_auto}

gl_auto is available in the version 3.0 release of the well-established MORGAN package [22, 32, 41]. The program produces samples of IVs consistent with the observed pedigree and genotype data alone; no phenotype data is used.gl_auto produces joint samples across loci by exact sampling when analyzing small pedigrees, and uses MCMC methods for large pedigrees. The efficiency and accuracy of the MCMC methods used to create these samples have been demonstrated with both sparse microsatellite and dense single nucleotide polymorphism data $[22,32,41]$. Computational demands are minimized by sampling IVs instead of genotypes, making the approach feasible across a range of pedigree sizes, marker types, and missing data patterns [41]. This keeps computation times low: e.g. 100,000 iterations for a 24 -member pedigree with 22 markers took 5.78 cpu minutes using the Intel L5420 Xeon $2.50 \mathrm{GHz}$ processor used for all analyses described below.

As with any linkage analysis, the user must combine a set of assumptions concerning the pedigree and marker data. Pedigree structure, a meiotic marker map, population-specific allele frequencies, and genotypes (where observed) are required. Additionally, the user must specify how many IV samples to generate and save, the maximum number of meioses to consider for exact computation instead of MCMC sampling, and sampler details. Pedigree and marker data are assumed to be without error: pedigree structure is accurately defined, no genotype errors are present, and the marker map and allele frequencies are correct. Standard tools exist for validat- 
ing pedigree structure and genotyping errors, and should be used as an initial quality control check $[22,32]$. Meioses are assumed to be independent from each other; the chromosomes inherited by one sibling do not influence which chromosomes another inherits. We assume the absence of genetic interference and the independence of alleles at neighboring markers at the population level. Hence, the markers used for gl_auto must be thinned based on estimates of linkage disequilibrium or map position. Judicious marker thinning also reduces computation time without loss of linkage information [42] and improves mixing of the MCMC sampler. These data and assumptions work together such that any genotypic dependence observed in the data set is due to IBD within the pedigree.

gl_auto uses two block Gibbs samplers to sample IVs conditional on the observed pedigree and marker data [25]. The locus-sampler is a modified version of the Elston-Stewart [28] algorithm, where the IVs at a single locus are sampled based on genotype data and the current IVs at all other loci. The meiosis-sampler is based on the Lander-Green [26] algorithm, where IVs at all loci for a single meiosis are updated based on the current IVs at all other meioses. The meiosis-sampler can either update a single meiosis at a time, or sets of multiple meioses using the Baum algorithm $[32,43]$. Once all IVs have been sampled $[22,32]$, the result is called a realization. Sampling multiple meioses considerably improves MCMC mixing, and consequently the estimation of IVs. This is especially true for pedigrees with large sibships [32]. The ratio of locus- to meiosis-sampling events across iterations can be varied. This approach has comparable accuracy and computational requirements relative to other MCMC-based linkage analysis packages when markers are sparsely spaced, but considerable improvements in both speed and accuracy when dense markers are used [32, 41]. Greater mathematical detail on these samplers is available elsewhere $[22,39]$.

gl_auto reports IVs at all markers for all subjects for each of the many saved realizations. Each realization provides both maternal and paternal records for each subject in the pedigree. Each record contains the following: the name of the individual, the FGL or meiosis indicator at the unlinked null trait locus, the FGL or meiosis indicator at the first marker locus, the $N$ number of recombination events or changes along the chromosome, then a list of $N$ pairs of integers. These pairs of integers consist of the marker locus index where the change occurs, then the new FGL or meiosis indicator associated with the following chromosomal segment [25]. The output file is kept relatively compact by only recording where changes occur along a chromosome: e.g. 2,000 realizations of IVs for a pedigree of 24 individuals was $2.4 \mathrm{MB}$.

\section{IBDgraph}

IBDgraph reads the IV sample generated by gl_auto to identify equivalencies within and between realizations, using computational and mathematical techniques previously described $[22,44]$. Two non-identical realizations are equivalent if the patterns of IBD sharing differ only with respect to the FGLs shared. Equivalence is determined by efficient strong hash coding; if two IBD graphs share the same hash code, they are equivalent. For a given marker, the observed genotypes and pedigree structure dramatically limit the number of possible IBD graphs. Similarly, across a chromosome, changes to IBD graphs are caused by rare recombination events. This means large segments of a chromosome are equivalent in a given realization. Across realizations, IBDgraph can find equivalence classes across all markers, a single marker, or a range of markers. Equivalence classes are listed, recording the number of realizations within the class, followed by a list of the realizations within each equivalence class. Within a single realization, IBDgraph will identify and list the marker intervals without recombination events. The user is therefore able to quickly evaluate the patterns of IBD sharing at a given marker/range; e.g. it took only $16 \mathrm{cpu}$ seconds to identify equivalence classes across 2,000 realizations across 31 markers in a 24-member pedigree. Any calculations that use IBD sharing patterns within a pedigree may be done on one representative of each equivalence class, rather than on every sampled marker/region/realization. The final calculation is then based on the weighted sum across equivalence classes, with weights proportional to the size of the class. This can dramatically reduce computation times; e.g. for the marker with the greatest evidence of linkage in the example described below, LOD score calculations based on equivalence classes were $\sim 10$ times faster than a singlemarker computation using the original data.

\section{Example: Inheritance Vectors Simplify the Interpretation of Complex Trait Linkage Results}

In this section, we illustrate how gl_auto and IBDgraph may be used to identify an IBD segment driving a linkage signal for a complex trait locus, as well as which 
Fig. 2. Pedigree with familial late-onset Alzheimer's disease. Circles are female, squares are male, filled symbols indicate affected individuals. Genotyped subjects are in bold. Beneath each subject is their age of onset (affected) or the last age at which the subject was known to be unaffected.

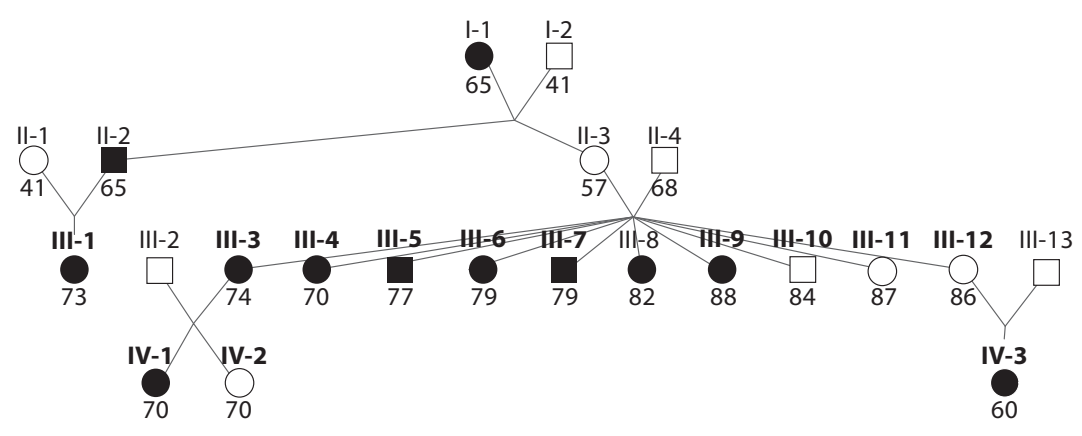

relatives are carriers. We use familial late-onset $\mathrm{AD}$ as an example phenotype because it has evidence of phenotypic, genetic, and allelic heterogeneity. Family history is a key risk factor for $\mathrm{AD}[45,46]$. Any one of hundreds of different rare variants in three genes [47] can cause rare early-onset $\mathrm{AD}$ (age $<65$ years), and their large functional effects have illuminated biochemical pathways underlying AD [48]. Identification of relevant genes for lateonset $\mathrm{AD}$ has been more difficult: only the apolipoprotein $\mathrm{E}$ gene $(A P O E)$ has been unequivocally established as a locus with relatively strong effect in both late-onset [49] and early-onset AD [50, 51].

For this example, we first detected a linkage signal by performing parametric multipoint linkage analysis on a single moderately large pedigree, shown in figure 2 . Genome-scan markers (ABI Prism Linkage Mapping set, Version 2.5, MD10, http://www.appliedbiosystems.com) were genotyped on subjects shown in bold in figure 2 . Missing genotype data among founders and imperfectly informative marker data reduce our power to detect linkage [3]. Analysis of multiple markers helps constrain the possible values for these missing genotypes [32]. We therefore applied a multipoint approach using IVs estimated only from all markers for all members of the pedigree, avoiding errors caused by trait model misspecification that are unique to multipoint analysis [52, 53]. For each class at each marker, we treated each FGL as a unique and equifrequent allele. This flexible multipoint strategy does not dictate the use of a particular linkage statistic [2, 24]. We chose parametric linkage analysis for this example because the approach is powerful when an appropriate trait model is used [2], although it will not suit all traits or data sets. We applied a previously published model of AD risk [54] using a modified version of LIPED [55]. We modeled a rare dominant $\mathrm{AD}$ risk variant with an age-dependent penetrance function, which helps to accommodate the censored phenotype data typical in this late-onset disease. We also included the number of $A P O E$ risk alleles as a covariate influencing sporadic risk of $\mathrm{AD}$, to allow for the influence of a known and important genetic risk factor. Although we do not expect any model to perfectly capture AD risk, the modeled mode of inheritance is plausible for this example; this level of model misspecification does not prevent a valid test $[3$, $56,57]$.

Our strongest linkage signal occurred on chromosome 9, centered on marker D9S175. IBDgraph collapsed the 2,000 sampled IVs at D9S175 into just three equivalence classes. The two largest equivalence classes representing $97.7 \%$ of realizations had a maximum $\mathrm{LOD}=2.36$ at recombination fraction $\theta=0.001$, while the third class had a maximum LOD $=1.62$ at $\theta=0.001$. This signal on $9 \mathrm{q} 21.13$ is consistent with the suggestive findings of a large and independent $\mathrm{AD}$ study ([58] and references therein), motivating further interrogation of the region.

Figures 3 and 4 illustrate patterns of IBD sharing among all affected relatives for whom DNA samples are available. We focus on IBD sharing among these subjects because the majority of a linkage signal is driven by affected subjects, and these subjects are available for future sequencing or genotyping studies. These figures were the product of plotIBD [59], a Perl script that takes gl_auto and IBDgraph output to produce commands for the open source graphing utility gnuplot [60]. These commands produce images comparable to what the CompareLinkage software generates from data formats meeting the requirements for the Merlin analysis package [61], which is limited to the analysis of small pedigrees. plotIBD offers both ease and flexibility: the user can pipe the commands directly into gnuplot, or exert greater 


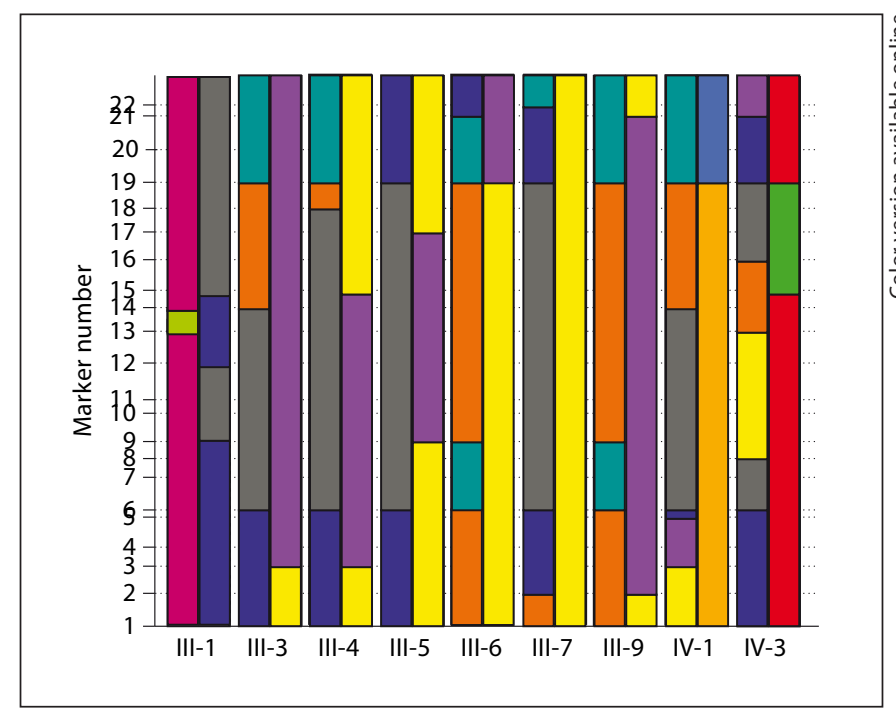

Fig. 3. Patterns of identity by descent sharing among affected relatives with DNA available, as defined by a single MCMC realization of chromosome 9 data. The maternal (paternal) chromosome is drawn to the left (right) for each subject. Blocks inherited from the same founder chromosome are filled with the same color. Tic marks are proportional to marker map distance.

control by adjusting the graphing parameters (font size, page orientation, etc.).

Figure 3 illustrates patterns of IBD sharing across chromosome 9, as represented in a single gl_auto realization. Each chromosome is broken into color-coded IBD segments. Subjects sharing the same color at a marker position have inherited that locus IBD from a common ancestor, according to a realization drawn from IBDgraph equivalence class 1 . The break points between IBD segments occur between markers; if a change is illustrated at marker 15, the causal recombination event is inferred to have occurred between markers 14 and 15 . Although sib pairs share an average of $50 \%$ of their genome IBD, figure 3 shows the variability in estimated IBD across chromosome 9 in a single MCMC realization. For example, notice that III- 4 and III- 9 share both alleles IBD from marker 21 through the end of the chromosome, but no alleles IBD between markers 15 and 18 .

Figure 4 illustrates patterns of IBD sharing across realizations at a single locus, D9S175 (fig. 3 marker 11). The classes are labeled along the y-axis in descending order of weight, so that class 1 is the equivalence class representing the most realizations. Figure 4 shows that siblings III3, III-4, III-5, and III-7 share their maternal allele IBD

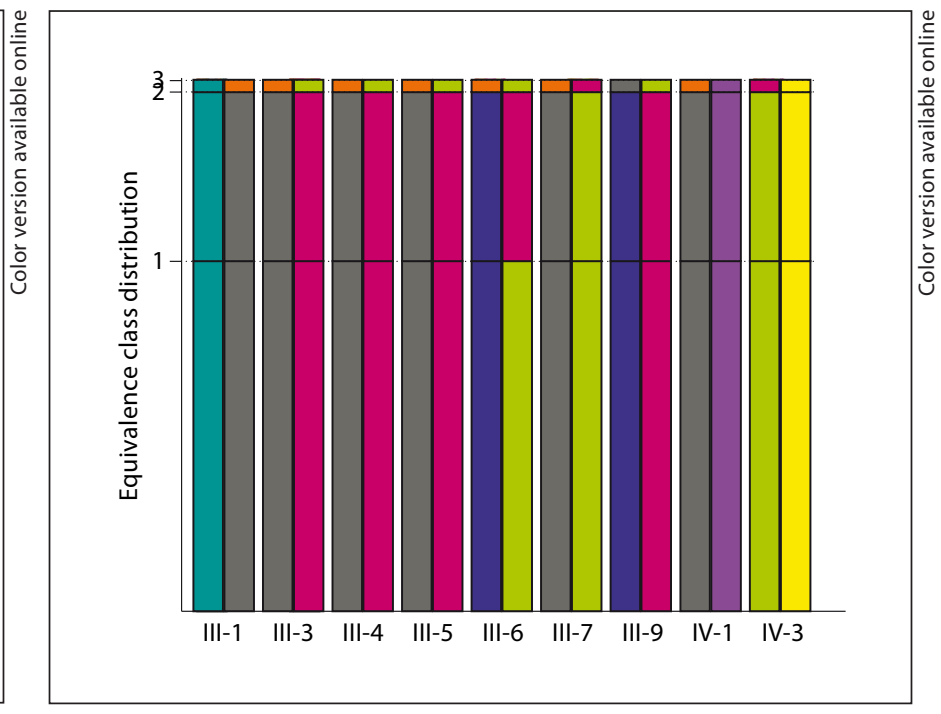

Fig. 4. Patterns of identity by descent sharing among affected relatives with DNA available, at D9S175 across all MCMC realizations. The maternal (paternal) chromosome is drawn to the left (right) for each subject. Blocks inherited from the same founder chromosome are filled with the same color. Along the y-axis, equivalence classes are drawn in proportion to their probability. The solid black pattern (online: grey) represents the IBD segment driving our linkage signal.

across all equivalence classes, while III- 6 and III-9 do not. If we assume an $\mathrm{AD}$ locus truly exists beneath the linkage signal on chromosome 9 , these results provide evidence of genetic and/or allelic heterogeneity of $\mathrm{AD}$ within this family: multiple affected subjects must carry the linked allele IBD in order to generate such a linkage signal, but the signal would be stronger if all affected subjects carried the same AD allele IBD (LOD $=\sim 3-5$ at $\theta=0$ ) [62].

We can use gl_auto results to identify probable carriers of the IBD segment co-segregating with the linkage signal on chromosome 9. Because we care only about this trait locus, we focus on IBD within the relatively small linkage region, rather than the whole chromosome or genome. This IBD segment is tagged by a multimarker haplotype, but its marker alleles and boundaries are not obvious from the linkage signal alone. Within a linkage region, we propose selecting the IBD segment that is most common among affected relatives, then testing whether this segment can explain the observed linkage signal. In this case, we used the evidence for linkage to identify a haplotype that appeared to be overshared among affected subjects. Individually, markers 8-13 (fig. 3) each had maximum LOD scores at the smallest possible recombination fraction considered $(\theta=0.001)$, so we chose the 
central four markers to build our haplotype. This haplotype corresponds to the IBD segment shared by III-1, III3, III-4, III-5, III-7, and IV-1 in figure 4. Parametric linkage analysis using the same trait model as before and a diallelic indicator of presence/absence of this haplotype recreated the linkage signal $(\mathrm{LOD}=2.07$ at $\theta=0.001$ ), suggesting that the haplotype tags the chromosomal segment generating our linkage signal. One could explore the boundaries of the shared IBD segment by comparing equivalence classes at the markers flanking the linkage signal, perhaps even assign probabilities to those boundaries. This would be most helpful when analyzing dense marker data, as they would offer greater resolution. The next step towards gene identification involves characterizing sequence variation within the linked region. If we assumed a single-gene model for AD risk, subjects III-1 and IV-3 would be a naïve choice of subjects for targeted sequencing analysis because they have the most distant possible relationship among affected subjects in this pedigree. More distant relatives share fewer and smaller IBD regions, which helps restrict the number of shared variants within the linkage region. Any rare variants with predicted functional effects that were shared by III-1 and IV-3 would then be candidate AD loci. However, as shown in figure 3, these two subjects share nothing IBD within the linkage region near D9S175. Sequencing of this region confirms the prediction within figure 3: no rare variants with predicted functional effects were shared by III-1 and IV-3. By performing linkage analysis and identifying regions of interest beforehand, we can identify a pair of affected subjects that shared the linked haplotype IBD with high probability, thereby increasing the odds of discovering an $\mathrm{AD}$ locus.

\section{Conclusions}

Modern computing algorithms and the estimation of IV equivalence classes allow us to examine IBD sharing patterns in large pedigrees and dense data sets with greater speed and precision than was previously possible. The MORGAN v3.0 program gl_auto provides samples of IVs with many uses. As shown in our AD example, we can estimate and explore IBD sharing patterns within a large pedigree with incomplete marker data. Within a linkage region, this can identify subjects for subsequent sequencing analyses. Identification of genetic variants shared by carriers of the linked IBD segment may then lead to an underlying trait locus, as illustrated in a recent study of a cardiovascular quantitative trait [9]. It is also possible to use gl_auto and IBDgraph to: (1) force software developed for single-marker analyses to perform multipoint analysis; (2) perform different linkage analyses on identical data using other statistics, trait models [63], or phenotypes; (3) estimate empirical p values (e.g. for variancecomponents analyses [64]); and (4) probabilistically phase and infer genotypes within a pedigree [65]. We may also share gl_auto realizations with a collaborator without sharing raw genotype data, which can help keep samples anonymous and unidentifiable. The companion programs IBDgraph and plotIBD ease the identification of patterns within this data, making the interpretation of complex trait linkage analyses a more manageable and feasible endeavor.

\section{Acknowledgements}

Financial support was provided by National Institute of Health (NIH) grants AG005136, AG000258, and GM046255.

\section{References}

1 Morton NE: Sequential tests for the detection of linkage. Am J Hum Genet 1955;7:277-318.

2 Kruglyak L, Daly MJ, Reeve-Daly MP, Lander ES: Parametric and nonparametric linkage analysis: A unified multipoint approach. Am J Hum Genet 1996;58:1347-1363.

-3 Terwilliger JD, Goring HH: Gene mapping in the 20th and 21st centuries: Statistical methods, data analysis, and experimental design. Human Biology 2000;72:63-132.

4 Botstein D, Risch N: Discovering genotypes underlying human phenotypes: Past successes for mendelian disease, future ap- proaches for complex disease. Nat Genet 2003;33:228-237.

5 Almasy L, Blangero J: Human QTL linkage mapping. Genetica 2009;136:333-340.

6 Ng SB, Nickerson DA, Bamshad MJ, Shendure $\mathrm{J}$ : Massively parallel sequencing and rare disease. Hum Mol Genet 2010;19:R119-R124.

7 Riazuddin S, Castelein CM, Ahmed ZM, Lalwani AK, Mastroianni MA, Naz S, Smith TN, Liburd NA, Friedman TB, Griffith AJ, Wilcox ER: Dominant modifier DFNM1 suppresses recessive deafness DFNB26. Nat Genet 2000;26:431-434. $\checkmark 8$ Daw EW, Chen SN, Czernuszewicz G, Lombardi R, Lu Y, Ma J, Roberts R, Shete S, Marian AJ: Genome-wide mapping of modifier chromosomal loci for human hypertrophic cardiomyopathy. Hum Mol Genet 2007;16: 2463-2471.

$>9$ Rosenthal EA, Ronald J, Rothstein J, Rajagopalan R, Ranchalis J, Wolfbauer G, Albers JJ, Brunzell JD, Motulsky AG, Rieder MJ, Nickerson DA, Wijsman EM, Jarvik GP: Linkage and association of phospholipid transfer protein activity to LASS4. J Lipid Res 2011; 52:1837-1846. 
10 Guerreiro RJ, Baquero M, Blesa R, Boada M, Bras JM, Bullido MJ, Calado A, Crook R, Ferreira C, Frank A, Gomez-Isla T, Hernandez I, Lleo A, Machado A, Martinez-Lage P, Masdeu J, Molina-Porcel L, Molinuevo JL, Pastor P, Perez-Tur J, Relvas R, Oliveira CR, Ribeiro MH, Rogaeva E, Sa A, Samaranch L, Sanchez-Valle R, Santana I, Tarraga L, Valdivieso F, Singleton A, Hardy J, Clarimon J: Genetic screening of Alzheimer's disease genes in Iberian and African samples yields novel mutations in presenilins and APP. Neurobiol Aging 2010;31:725-731.

-11 Campion D, Dumanchin C, Hannequin D, Dubois B, Belliard S, Puel M, Thomas-Anterion C, Michon A, Martin C, Charbonnier F, Raux G, Camuzat A, Penet C, Mesnage V, Martinez M, Clerget-Darpoux F, Brice A, Frebourg T: Early-onset autosomal dominant Alzheimer disease: prevalence, genetic heterogeneity, and mutation spectrum. Am J Hum Genet 1999;65:664-670.

-12 Bernardi L, Tomaino C, Anfossi M, Gallo M, Geracitano S, Puccio G, Colao R, Frangipane F, Mirabelli M, Smirne N, Maletta RG, Bruni AC: Late onset familial Alzheimer's disease: novel presenilin 2 mutation and PS1 E318G polymorphism. J Neurol 2008;255:604-606.

- 13 Gomez-Isla T, Growdon WB, McNamara MJ, Nochlin D, Bird TD, Arango JC, Lopera F, Kosik KS, Lantos PL, Cairns NJ, Hyman BT: The impact of different presenilin 1 and presenilin 2 mutations on amyloid deposition, neurofibrillary changes and neuronal loss in the familial Alzheimer's disease brain - evidence for other phenotype-modifying factors. Brain 1999;122:1709-1719.

-14 McClellan J, King MC: Genetic heterogeneity in human disease. Cell 2010;141:210-217.

15 Hobbs HH, Russell DW, Brown MS, Goldstein JL: The LDL receptor locus in familial hypercholesterolemia - mutational analysis of a membrane-protein. Annu Rev Genet 1990;24:133-170.

- 16 Wilund KR, Yi M, Campagna F, Arca M, Zuliani G, Fellin R, Ho YK, Garcia JV, Hobbs $\mathrm{HH}$, Cohen JC: Molecular mechanisms of autosomal recessive hypercholesterolemia. Hum Mol Genet 2002;11:3019-3030.

17 Wijsman EM, Amos CI: Genetic analysis of simulated oligogenic traits in nuclear and extended pedigrees: Summary of GAW10 contributions. Genet Epidemiol 1997;14:719735.

18 Wright AF, Carothers AD, Pirastu M: Population choice in mapping genes for complex diseases. Nat Genet 1999;23:397-404.

-19 Manolio TA, Collins FS, Cox NJ, Goldstein DB, Hindorff LA, Hunter DJ, McCarthy MI, Ramos EM, Cardon LR, Chakravarti A, Cho JH, Guttmacher AE, Kong A, Kruglyak L, Mardis E, Rotimi CN, Slatkin M, Valle D, Whittemore AS, Boehnke M, Clark AG, Eichler EE, Gibson G, Haines JL, Mackay TFC, McCarroll SA, Visscher PM: Finding the missing heritability of complex diseases. Nature 2009;461:747-753.
20 Marchani EE, Bird TD, Steinbart EJ, Rosenthal E, Yu CE, Schellenberg GD, Wijsman EM: Evidence for three loci modifying ageat-onset of Alzheimer's disease in early-onset PSEN2 families. Am J Med Genet B 2010; 153B:1031-1041.

21 Hasstedt SJ, Scott BT, Callas PW, Vossen CY, Rosendaal FR, Long GL, Bovill EG: Genome scan of venous thrombosis in a pedigree with protein $\mathrm{c}$ deficiency. J Thromb Haemost 2004;2:868-873.

22 Thompson EA: The structure of genetic linkage data: from LIPED to 1M SNPs. Hum Hered 2011;71:86-96.

23 Thomas A, Camp NJ, Farnham JM, AllenBrady K, Cannon-Albright LA: Shared genomic segment analysis. Mapping disease predisposition genes in extended pedigrees using SNP genotype assays. Ann Hum Genet 2008;72:279-287.

24 Sobel E, Lange K: Descent graphs in pedigree analysis: Applications to haplotyping, location scores, and marker-sharing statistics. Am J Hum Genet 1996;58:1323-1337.

25 MORGAN: A package for Markov chain Monte Carlo in genetic analysis (version 3.0), 2009. http://www.stat.washington.edu/thompson/ Genepi/MORGAN/Morgan.shtml.

26 Lander ES, Green P: Construction of multilocus genetic maps in humans. P Natl Acad Sci USA 1987;84:2363-2367.

27 Donnelly KP: The probability that related individuals share some section of genome identical by descent. Theor Popul Biol 1983; 23:34-63.

28 Elston RC, Stewart J: A general model for the genetic analysis of pedigree data. Hum Hered 1971;21:523-542.

29 Gao GM, Allison DB, Hoeschele I: Haplotyping methods for pedigrees. Hum Hered 2009;67:248-266.

30 Lange K, Sobel E: A random walk method for computing genetic location scores. Am J Hum Genet 1991;49:1320-1334.

31 Sobel E, Sengul H, Weeks DE: Multipoint estimation of identity-by-descent probabilities at arbitrary positions among marker loci on general pedigrees. Hum Hered 2001;52:121131.

32 Tong LP, Thompson E: Multilocus lod scores in large pedigrees: Combination of exact and approximate calculations. Hum Hered 2008; 65:142-153.

-33 Thompson EA, Lin S, Olshen AB, Wijsman EM: Monte Carlo segregation and linkage analysis of a large hypercholesterolemia pedigree. Genet Epidemiol 1993;10:677-682.

34 Thompson EA: Monte Carlo likelihood in genetic mapping. Stat Sci 1994;9:355-366.

35 Thompson EA, Heath SC: Estimation of conditional multilocus gene identity among relatives; in Seillier-Moseiwitch F, Donnelly P, Waterman M (eds): Statistics in Molecular Biology and Genetics: Selected Proceedings of the 1997 Joint AMS-IMSSIAM Summer Conference on Statistics in Molecular Biology. Hayward, CA, Institute of Mathematical Statistics, 1999, vol 33, pp 93-113.

36 Thompson EA: MCMC estimation of multi-locus genome sharing and multipoint gene location scores. Int Stat Rev 2000;68:53-73.

37 Heath SC: Markov chain Monte Carlo segregation and linkage analysis for oligogenic models. Am J Hum Genet 1997;61:748-760.

38 Daw EW, Heath SC, Wijsman EM: Multipoint oligogenic analysis of age-at-onset data with applications to Alzheimer's disease pedigrees. Am J Hum Genet 1999;64:839851.

39 Thompson EA: Statistical inferences from genetic data on pedigrees. Beachwood, Ohio, IMS, 2000.

40 Thompson EA: MCMC in the analysis of genetic data on pedigrees; in Kendall WS, Wang JS, Liang F (eds): Markov Chain Monte Carlo: Innovations and Applications. Singapore, World Scientific Publishing Company, 2005.

41 Wijsman EM, Rothstein JH, Thompson EA: Multipoint linkage analysis with many multiallelic or dense diallelic markers: MCMC provides practical approaches for genome scans on general pedigrees. Am J Hum Genet 2006;79:846-858.

42 Wilcox MA, Pugh EW, Zhang H, Zhong X, Levinson DF, Kennedy GC, Wijsman EM: Comparison of single-nucleotide polymorphisms and microsatellite markers for linkage analysis in the COGA and simulated data sets for Genetic Analysis Workshop 14:Presentation groups 1, 2, and 3. Genet Epidemiol 2005;29 (suppl 1):S7-S28.

43 Baum LE: An inequality and associated maximization technique in statistical estimation for probabilistic functions on Markov processes; in Shisha O (ed): Inequalitiesiii; Proceedings of the Third Symposium on Inequalities University of California Los Angeles, 1969. New York, Academic Press, 1972, pp 1-8.

44 Koepke HA, Thompson EA: Efficient testing operations on dynamic graph structures using strong hash functions: Technical report \#567, Department of Statistics, University of Washington, available at http://www.stat. washington.edu/research/reports/2010/ tr567.pdf, 2010.

45 Lautenschlager NT, Cupples LA, Rao VS, Auerbach SA, Becker R, Burke J, Chui H, Duara R, Foley EJ, Glatt SL, Green RC, Jones R, Karlinsky H, Kukull WA, Kurz A, Larson EB, Martelli K, Sadovnick AD, Volicer L, Waring SC, Growdon JH, Farrer LA: Risk of dementia among relatives of Alzheimer's disease patients in the MIRAGE study: What is in store for the oldest old? Neurology 1996;46: 641-650.

46 Mayeux R, Sano M, Chen J, Tatemichi T, Stern Y: Risk of dementia in 1st-degree relatives of patients with Alzheimers-disease and related disorders. Arch Neurol 1991;48: 269-273. 
47 Cruts M: Alzheimer disease \& frontotemporal dementia mutation database, 2010. http:// www.molgen.ua.ac.be/ADMutations and http://www.molgen.ua.ac.be/FTDMutations.

48 Tanzi RE, Bertram L: Twenty years of the Alzheimer's disease amyloid hypothesis: A genetic perspective. Cell 2005;120:545555.

-49 Corder EH, Saunders AM, Strittmatter WJ, Schmechel DE, Gaskell PC, Small GW, Roses AD, Haines JL, Pericak-Vance MA: Gene dose of apolipoprotein E type 4 allele and the risk of Alzheimer's disease in late onset families. Science 1993;261:921-923.

50 Pastor P, Roe C, Villegas A, Bedoya G, Chakraverty S, Garcia G, Tirado V, Norton J, Rios S, Martinez M, Kosik K, Lopera F, Goate A: Apolipoprotein E e4 modifies Alzheimer's disease onset in an E280A PS1 kindred. Ann Neurol 2003;54:163-169.

51 Wijsman EM, Daw EW, Yu X, Steinbart EJ, Nochlin D, Bird TD, Schellenberg G: APOE and other loci affect age-at-onset in $\mathrm{Al}$ zheimer's disease families with PS2 mutation. Am J Med Genet B 2005;132B:14-20.

- 52 Risch N, Giuffra L: Model misspecification and multipoint linkage analysis. Hum Hered 1992;42:77-92.

53 Göring HHH, Terwilliger JD: Linkage analysis in the presence of errors IV: Joint pseu- domarker analysis of linkage and/or linkage disequilibrium on a mixture of pedigrees and singletons when the mode of inheritance cannot be accurately specified. Am J Hum Genet 2000;66:1310-1327.

54 Levy-Lahad E, Wasco W, Poorkaj P, Romano DM, Oshima J, Pettingell WH, Yu CE, Jondro PD, Schmidt SD, Wang K, Crowley AC, Fu YH, Guenette SY, Galas D, Nemens E, Wijsman EM, Bird TD, Schellenberg GD, Tanzi RE: Candidate gene for the chromosome 1 familial Alzheimer's disease locus. Science 1995;269:973-977.

55 Ott J: Estimation of the recombination fraction in human pedigrees: Efficient computation of the likelihood for human linkage studies. Am J Hum Genet 1974;26:588-597.

56 Clerget-Darpoux F, Bonaiti-Pellie C, Hochez $\mathrm{J}$ : Effects of misspecifying genetic parameters in lod score analysis. Biometrics 1986;42: 393-399.

57 Williamson JA, Amos CI: On the asymptotic-behavior of the estimate of the recombination fraction under the null hypothesis of no linkage when the model is misspecified. Genet Epidemiol 1990;7:309-318.

58 Hamshere ML, Holmans PA, Avramopoulos D, Bassett SS, Blacker D, Bertram L, Wiener H, Rochberg N, Tanzi RE, Myers A, Vrieze FWD, Go R, Fallin D, Lovestone S, Hardy J,
Goate A, O’Donovan M, Williams J, Owen MJ: Genome-wide linkage analysis of 723 affected relative pairs with late-onset Alzheimer's disease. Hum Mol Genet 2007;16: 2703-2712.

59 plotIBD.pl: http://faculty.washington.edu/ wijsman/software.shtml, 2011.

60 Gnuplot: http://gnuplot.Info/, 2009.

61 Leykin I, Hao K, Cheng JS, Meyer N, Pollak MR, Smith RJ, Wong WH, Rosenow C, Li C: Comparative linkage analysis and visualization of high-density oligonucleotide SNP array data. BMC Genet 2005;6:7.

62 Ploughman LM, Boehnke M: Estimating the power of a proposed linkage study for a complex genetic trait. Am J Hum Genet 1989;44: 543-551.

63 Logue MW, Vieland VJ: A new method for computing the multipoint posterior probability of linkage. Hum Hered 2004;57:9099.

64 Marchani EE, Di Y, Choi Y, Cheung C, Su M, Boehm F, Thompson EA, Wijsman EM: Contrasting IBD estimators, association studies, and linkage analysis using the Framingham Heart Study. BMC Proceedings 2009;3:S102.

65 Cheung CY, Thompson EA, Wijsman EM: In silico genotype imputation on large pedigrees. Genet Epidemiol 2010;34:919. 